\title{
SNP-based real-time pyrosequencing as a sensitive and specific tool for identification and differentiation of Rickettsia species in Ixodes ricinus ticks
}

\author{
Elisabeth Janecek, Sabine Streichan and Christina Strube*
}

\begin{abstract}
Background: Rickettsioses are caused by pathogenic species of the genus Rickettsia and play an important role as emerging diseases. The bacteria are transmitted to mammal hosts including humans by arthropod vectors. Since detection, especially in tick vectors, is usually based on PCR with genus-specific primers to include different occurring Rickettsia species, subsequent species identification is mainly achieved by Sanger sequencing. In the present study a real-time pyrosequencing approach was established with the objective to differentiate between species occurring in German Ixodes ticks, which are R. helvetica, $R$. monacensis, $R$. massiliae, and $R$. felis. Tick material from a quantitative real-time PCR (qPCR) based study on Rickettsia-infections in I. ricinus allowed direct comparison of both sequencing techniques, Sanger and real-time pyrosequencing.
\end{abstract}

Methods: A sequence stretch of rickettsial citrate synthase $(\mathrm{g} / \mathrm{t} A)$ gene was identified to contain divergent single nucleotide polymorphism (SNP) sites suitable for Rickettsia species differentiation. Positive control plasmids inserting the respective target sequence of each Rickettsia species of interest were constructed for initial establishment of the real-time pyrosequencing approach using Qiagen's PSQ 96MA Pyrosequencing System operating in a 96-well format. The approach included an initial amplification reaction followed by the actual pyrosequencing, which is traceable by pyrograms in real-time. Afterwards, real-time pyrosequencing was applied to 263 Ixodes tick samples already detected Rickettsia-positive in previous QPCR experiments.

Results: Establishment of real-time pyrosequencing using positive control plasmids resulted in accurate detection of all SNPs in all included Rickettsia species. The method was then applied to 263 Rickettsia-positive Ixodes ricinus samples, of which 153 (58.2\%) could be identified for their species (151 R. helvetica and 2 R. monacensis) by previous custom Sanger sequencing. Real-time pyrosequencing identified all Sanger-determined ticks as well as 35 previously undifferentiated ticks resulting in a total number of 188 (71.5\%) identified samples. Pyrosequencing sensitivity was found to be strongly dependent on gltA copy numbers in the reaction setup. Whereas less than $10^{1}$ copies in the initial amplification reaction resulted in identification of $15.1 \%$ of the samples only, the percentage increased to $54.2 \%$ at $10^{1}-10^{2}$ copies, to $95.6 \%$ at $>10^{2}-10^{3}$ copies and reached $100 \%$ samples identified for their Rickettsia species if more than $10^{3}$ copies were present in the template.

(Continued on next page)

\footnotetext{
* Correspondence: christina.strube@tiho-hannover.de

Institute for Parasitology, University of Veterinary Medicine Hannover, Buenteweg 17, Hannover 30559, Germany
} 
(Continued from previous page)

Conclusions: The established real-time pyrosequencing approach represents a reliable method for detection and differentiation of Rickettsia spp. present in I. ricinus diagnostic material and prevalence studies. Furthermore, the method proved to be faster, more cost-effective as well as more sensitive than custom Sanger sequencing with simultaneous high specificity.

Keywords: Rickettsia helvetica, Rickettsia monacensis, Rickettsia massiliae, Rickettsia felis, Ixodes ricinus, Diagnostic, Sequencing

\section{Background}

Rickettsia spp. are Gram-negative, obligate intracellular bacteria with pleomorphic appearance [1]. The life cycle involves arthropod vectors like ticks, fleas, lice and mites, which are of great importance for the natural retention of the pathogen [2]. Tick-borne rickettsioses are originating from species belonging to the spotted fever group [3]. The most abundant European tick species Ixodes ricinus serves as vector for the potentially pathogenic Rickettsia species $R$. helvetica and $R$. monacensis [4-7]. Additionally, $R$. massiliae as well as $R$. felis has been detected in I. ricinus in one case each [5]. However, no vector competences are proven for those species.

$R$. helvetica is connected to "summer flu", a feverish infection without rash $[5,8]$, but also meningitis and perimyocarditis $[9,10]$. By contrast, $R$. monacensis, $R$. massiliae, and $R$. felis cause the classical form of spotted fever [5]. Even though Boretti et al. [11] describe a species-specific real-time PCR for $R$. helvetica based on the $23 \mathrm{~S}$ rRNA gene, Rickettsia detection in Ixodes ticks is mainly based on conventional or quantitative real-time PCR (qPCR) with genus- but not species-specific primers to include the different Rickettsia species followed by species differentiation via DNA sequencing with the classical chain termination by Sanger.

A more recent sequencing technology is pyrosequencing, which is based on the principle that nucleotide incorporation in a DNA strand is accompanied by release of pyrophosphate. This is converted into ATP by ATP sulfurylase in the presence of adenosine $5^{\prime}$ phosphosulfate. ATP, in turn, drives conversion of luciferin to oxyluciferin that emits visible light. This light signal is visualized as a peak, whose height is proportional to the number of nucleotides incorporated. To prevent incorrect detection, unincorporated nucleotides and ATP are degraded by apyrase before another nucleotide is added.

In the present study, a single nucleotide polymorphism (SNP)-based real-time pyrosequencing approach was evaluated as an in-lab diagnostic tool for detection and differentiation of Rickettsia spp. in I. ricinus. The used PSQ 96MA Pyrosequencing System (Qiagen, formerly Biotage) displays the peaks of incorporated nucleotides in real time in the so called pyrogram.

\section{Results}

\section{Establishment of real-time pyrosequencing}

In real-time generated pyrograms revealed that in all SNP positions (Figure 1) of all Rickettsia positive control plasmids ( $R$. helvetica, R. massiliae, and R. monacensis, and two $R$. felis plasmids) the correct nucleotide was incorporated exclusively (exemplarily see Figure $2 \mathrm{a}$ and $2 \mathrm{c}$ ), thus providing specific Rickettsia spp. differentiation. As evident from Figure 1, the first SNP position already delimits $R$. felis (nucleotide " $\mathrm{T}$ ") from the remaining species, which contain the nucleotide " $C$ " at this position. The fourth SNP is specific to $R$. monacensis and SNP 5 to $R$. massiliae, whereas $R$. helvetica-specificity is based on the combination of SNP 1 and 6.

\section{Pyrosequencing of Rickettsia-positive tick DNA}

Whilst Sanger sequencing identified 151 (57.4\%) and 2 (0.8\%) out of the 263 available ticks to be infected with $R$. helvetica and $R$. monacensis, respectively, the established real-time pyrosequencing confirmed all Sanger-identified tick samples and additionally detected $R$. helvetica in $35(31.8 \%)$ of 110 previously unidentified samples. So the overall real-time pyrosequencing Rickettsia differentiation rate was $71.5 \%$ (188/263), whereas custom Sanger sequencing identified 58.2\% (153/263) tick samples only. This difference was statistically significant $(\mathrm{P}=0.002)$. Detailed information on statistical results is given in Table 1.

Sensitivity of pyrosequencing increased with gltA copy numbers: if $>10^{2}-10^{3}$ copies were present in $2 \mu \mathrm{l}$ template of the initial amplification reaction, the detection rate was $95.6 \%$ (43/45 tick samples), and reached $100 \%$ $(121 / 121)$ in presence of more than $10^{3}$ glt $A$ copies. Rickettsia species differentiation was possible only in $24.7 \%$ of the low-burden ticks, divided into a differentiation rate of $15.1 \%(11 / 73$ tick samples) in case of less than $10^{1}$ copies/template and $54.2 \%(13 / 24)$ in case of $10^{1}-10^{2}$ copies. All but two tick samples identified for their Rickettsia species by real-time pyrosequencing but not custom Sanger sequencing contained less than $10^{3} \mathrm{glt} A$ copies/2 $\mu$ l template. In 198 of the 263 samples (75.3\%), at least one SNP position showed a proportion of less than $100 \%$ for a specific nucleotide (exemplarily shown in Figure 2b). Detailed sensitivity comparison of both methods is shown in Table 1. 


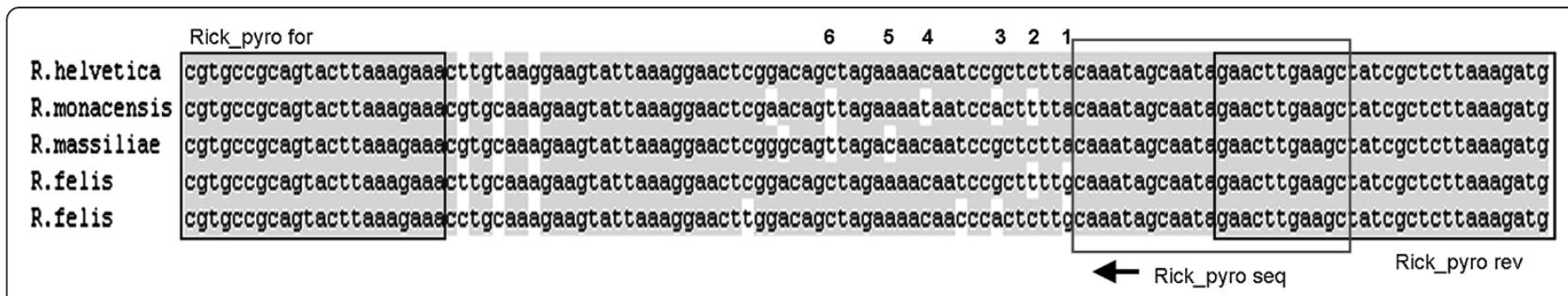

Figure 1 SNPs in the rickettsial gltA gene allowing species differentiation by real-time pyrosequencing. Used primers are marked by boxes. Note that Rick_pyro rev and Rick_pyro seq consist of complementary nucleotides and thus the lagging strand is sequenced. Detected SNPs are indicated by numbers. The displayed alignment is on GenBank accession numbers DQ910785 (R. helvetica), GQ925820 (R. monacensis), DQ459393 (R. massiliae), U33922 and GQ255903 (R. felis).

\section{Discussion}

Rickettsioses play an important role as emerging diseases, so there is a need for appropriate diagnostic tools. Our results show that real-time pyrosequencing is a specific and very sensitive diagnostic tool for differentiation between Rickettsia species occurring in I. ricinus in Europe.

Specificity of the established Rickettsia real-time pyrosequencing was validated by use of positive control plasmids containing the gltA target sequence of the individual Rickettsia species of interest. Even if detection of "exclusive" or other SNPs was not 100\% (cf. Figure 2b), the combination of the remaining SNPs enabled Rickettsia species differentiation. Application to diagnostic samples, which were DNA samples of I. ricinus ticks naturally infected with rickettsiae, showed superior sensitivity of real-time pyrosequencing compared to custom Sanger sequencing results from a previous study [12].

Not unexpectedly, real-time pyrosequencing sensitivity was strongly dependent on gltA copy numbers revealed by qPCR [12]. Low copy numbers $\left(\leq 10^{2}\right)$ in the initial amplification reaction often resulted in either weak or missing bands in gel electrophoresis with the consequence of insufficient template for subsequent pyrosequencing. Notably, all but two tick samples identified for their Rickettsia species by real-time pyrosequencing but not custom Sanger sequencing contained less than $10^{3} \mathrm{glt} A$ copies $/ 2 \mu \mathrm{l}$ template demonstrating that superior sensitivity of real-time pyrosequencing is due to successful sequencing of lower target copy numbers.

Detection of less than $100 \%$ for a specific nucleotide may be due to incorrect signaling, since double infections (two Rickettsia species in one tick) do not seem to occur [13] and have yet to be observed (upon request the manufacturer's technical support stated that $5 \%$ incorrect signaling is in the range of tolerance). Nevertheless, identification of the Rickettsia species ( $R$. helvetica in all cases) was possible based on the remaining SNP positions (see above).

\section{Conclusions}

We have developed the first real-time pyrosequencing approach for Rickettsia species. The method provides useful in-lab detection and species identification in diagnostic (tick) material as well as prevalence studies. Since rickettsioses gain attention as emerging diseases, there is a need for appropriate diagnostic tools. Real-time pyrosequencing proved to be faster, more cost-effective as well as more sensitive than custom Sanger sequencing. In detail, Rickettsia species identification by pyrosequencing usually takes about an hour, which includes preparation of samples as well as the actual sequencing whereas results of custom Sanger sequencing lasts at least until the next day. Additionally, the entire pyrosequencing procedure (initial PCR and pyrosequencing reaction itself) using Qiagen's PSQ 96MA sequencer does not exceed $1.50 €$ per sample. Thus, it is an optimal completion of studies detecting the genus Rickettsia but not individual species.

\section{Methods}

Selection of a target sequence stretch containing suitable SNPs

A sequence stretch of the rickettsial citrate synthase $(g l t A)$ gene was identified to contain divergent SNP sites suitable for species differentiation of $R$. helvetica, $R$. massiliae, $R$. monacensis, and $R$. felis. Thereby, SNP selection was based on sequences published in the GenBank database [accession nos. DQ910785, DQ131912, RHU59723, EU359285, EU359286, EU359287 (R. helvetica); GQ925820, GQ925822, DQ100163, EU665235, EU665236 (R. monacensis); DQ459393, DQ212705, region 1303919-1305247 of CP000683 (R. massiliae); as well as U33922, GQ255903, and region 1424392-1425699 of CP000053 (R. felis)].

\section{Positive control plasmids}

Positive control plasmids containing the respective target sequence of each included Rickettsia species was constructed using overlapping primers, whose sequences are listed in Table 2. PCR set up was as follows: $20.5 \mu \mathrm{l}$ 


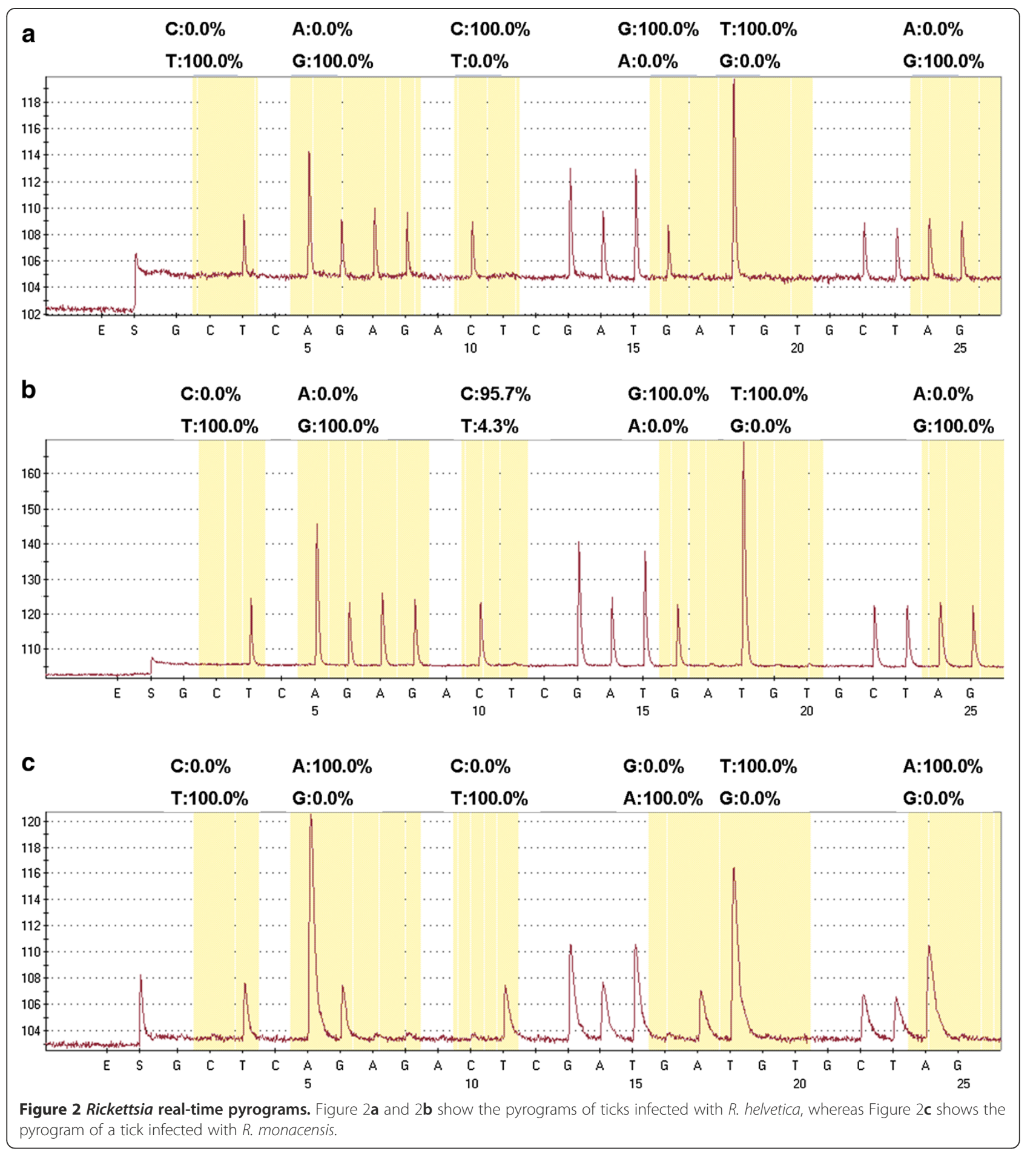

$\mathrm{ddH}_{2} \mathrm{O}$, each $0.5 \mu \mathrm{l}$ forward and reverse primer (10 pmol), $0.5 \mu \mathrm{ldNTPs}(10 \mathrm{mM}$ each), $2.5 \mu \mathrm{l} 10 \mathrm{x}$ buffer and $0.5 \mu \mathrm{l}$ Advantage 2 Polymerase Mix (Clontech). Cycling conditions consisted of $95^{\circ} \mathrm{C}$ for $1 \mathrm{~min}, 35$ cycles at $95^{\circ} \mathrm{C}$ for 30 sec, $55^{\circ} \mathrm{C}$ for $1 \mathrm{~min}$ and $72^{\circ} \mathrm{C}$ for $30 \mathrm{sec}$ followed by final elongation at $72^{\circ} \mathrm{C}$ for $10 \mathrm{~min}$. Amplification products were inserted into the $\mathrm{PCR}^{\circledR} 4-\mathrm{TOPO}{ }^{\circledR}$ vector (TOPO TA
Cloning ${ }^{\circledR}$ Kit for Sequencing, Life Technologies) and transformed into One Shot ${ }^{\circledR}$ TOP10 chemically competent E. coli (Life Technologies). Replicated plasmid DNA was extracted with the NucleoSpin ${ }^{\circledR}$ Plasmid kit (Macherey-Nagel) and accuracy of the rickettsial insert sequences was verified by sequencing (Seqlab Sequencing Laboratories). 
Table 1 Comparison of Sanger sequencing and real-time pyrosequencing

\begin{tabular}{|c|c|c|c|}
\hline \multicolumn{4}{|c|}{ Sensitivity } \\
\hline \multirow[t]{2}{*}{$\begin{array}{c}\text { Copy number in } \\
\text { initial template }(2 \mu \mathrm{l})\end{array}$} & $\begin{array}{c}\text { Sanger } \\
\text { Sequencing }\end{array}$ & $\begin{array}{c}\text { real-time } \\
\text { pyrosequencing }\end{array}$ & \multirow[t]{2}{*}{ P-value } \\
\hline & \multicolumn{2}{|c|}{$\begin{array}{c}\text { Number of identified tick } \\
\text { samples (\%) }\end{array}$} & \\
\hline$<10^{1}$ & $1 / 73(1.4)$ & $11 / 73(15.1)$ & $0.013^{*}$ \\
\hline $10^{1}-10^{2}$ & $5 / 24(8.3)$ & $13 / 24(54.2)$ & 0.180 \\
\hline$>10^{2}-10^{3}$ & $28 / 45(62.2)$ & 43/45 (95.6) & 0.239 \\
\hline$>10^{3}-10^{4}$ & $72 / 74(97.3)$ & $74 / 74(100)$ & 0.999 \\
\hline$>10^{5}-10^{5}$ & $42 / 42(100)$ & $42 / 42(100)$ & 1.000 \\
\hline$>10^{5}-10^{6}$ & 4/4 (100) & 4/4 (100) & 1.000 \\
\hline$>10^{6}$ & $1 / 1(100)$ & 1/1 (100) & 1.000 \\
\hline all samples & $153 / 263(58.2)$ & 188/263 (71.5) & $0.002^{*}$ \\
\hline
\end{tabular}

* Statistically significant.

\section{Primer design for pyrosequencing}

Primers for real-time-pyrosequencing were designed using the Pyrosequencing ${ }^{\mathrm{TM}}$ Assay Design software (version 1.0; Biotage). Primer positions within the target sequence and detected SNPs of the different Rickettsia species are shown in Figure 1. It should be noted that the pyrosequencing primer Rick_pyro seq sequences the lagging strand.

\section{Establishment of real-time pyrosequencing}

For initial establishment, each $2 \mu$ Rickettsia positive control plasmid DNA served as template in an initial

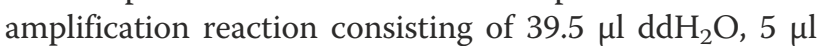
10x buffer, $0.5 \mu \mathrm{l}$ PerfectTaq Polymerase (5'PRIME), $1 \mu \mathrm{l}$ 5'-biotinylated Rick_pyro for, $1 \mu$ l Rick_pyro rev (10 pmol each; Sigma-Aldrich), and $1 \mu \mathrm{l}$ dNTPs (10 mM each). Thermocycling was as follows: $95^{\circ} \mathrm{C}$ for $3 \mathrm{~min}$ followed by 45 cycles of $95^{\circ} \mathrm{C}, 60^{\circ} \mathrm{C}$ and $72^{\circ} \mathrm{C}$ for each 30 seconds and final elongation at $72^{\circ} \mathrm{C}$ for 10 minutes. Products were checked by gel electrophoresis. Distinct bands at $115 \mathrm{bp}$ allowed further processing with the PSQ 96MA Pyrosequencing System (Qiagen), which operates in a 96 well format. First, $40 \mu \mathrm{l}$ biotinylated PCR product was immobilized to $4 \mu \mathrm{l}$ Streptavidin
Sepharose ${ }^{\mathrm{TM}}$ beads (GE Healthcare) with $40 \mu \mathrm{l}$ binding buffer $(10 \mathrm{mM} \mathrm{HCl}, 1 \mathrm{mM}$ EDTA, $2 \mathrm{M} \mathrm{NaCl}, 0.1 \%$ Tween 20, pH 7.6) for 10 minutes with vigorous shaking. The resulting complex was captured with the PyroMark Vacuum Prep Tool (Qiagen) and washed with 70\% ethanol for 5 seconds. Strand separation was achieved by treatment with denaturing buffer $(0.2 \mathrm{M} \mathrm{NaOH})$ for 5 seconds followed by 10 seconds washing with $10 \mathrm{mM}$ Tris-acetate buffer, pH 7.6. Afterwards, the biotinylated single strands were transferred into a PSQ 96 Plate Low containing $40 \mu \mathrm{l}$ annealing buffer $(20 \mathrm{mM}$ Tris-acetate, 2 $\mathrm{mM} \mathrm{MgAC}$ ) and $0.4 \mu \mathrm{M}$ sequencing primer (Rick_pyro seq). Following incubation for 2 minutes at $80^{\circ} \mathrm{C}$ using the PSQ 96 Sample Prep Thermoplate Low, required real-time pyrosequencing reagents (nucleotides as well as enzyme- and substrate mixtures; Pyro Mark ${ }^{\circledR}$ Gold Q96 Reagents, Qiagen) were added to the PSQ 96 Reagent Cartridge according to the volume information given by the instrument's software. The sequence to analyze entered into the PyroMark ID software was: $\mathrm{C} / \mathrm{T}$ AA A/G AG C/T GGATT G/A TT T/G TCTA G/A C. The nucleotide dispensation order to differentiate between the Rickettsia spp. included blank controls and was as follows: GCTCAGAGACTCGATGATGTGCATG (cf. Figure 2). Incorporated nucleotides were visualized in real-time pyrograms.

\section{Real-time pyrosequencing of Rickettsia-infected ticks}

To apply the established real-time pyrosequencing to diagnostic samples, I. ricinus tick DNA samples were evaluated. In a previous qPCR study, 363 questing I. ricinus DNA samples were found positive for Rickettsia spp. by qPCR [12], of which a subset of 263 samples was available for the present study. The samples comprised all developmental tick stages (larvae, nymphs as well as female and male adults) and included different numbers of glt $A$ copies per $2 \mu$ templates: 73 tick samples contained less than $10^{1}$ copies; 24 samples contained $10^{1}-10^{2}$ copies; 45 samples $>10^{2}-10^{3}$ copies; 74 samples $>10^{3}-10^{4}$ copies; 42 samples $>10^{5}-10^{5}$ copies; 4 samples $>10^{5}-10^{6}$ copies; and one sample contained $>10^{6}$ copies. Tick samples were amplified and

Table 2 Overlapping primers for Rickettsia positive control plasmids

\begin{tabular}{|c|c|c|}
\hline & Forward primer & Reverse primer \\
\hline R. helvetica & $\begin{array}{l}\text { 5'-CGTGCCGCAGTACTTAAAGAAACTTGTAAGGAAGTA } \\
\text { TTAAAGGAACTCGGACAGCTAGAAAACAATCCGC-3' }\end{array}$ & $\begin{array}{l}\text { 5'-CATCTTAAGAGCGATAGCTTCAAGTTCTATTGCT } \\
\text { ATTTGTAAGAGCGGATTGTITTCTAGCTGTCCGAG-3' }\end{array}$ \\
\hline R. monacensis & $\begin{array}{l}\text { 5'-CGTGCCGCAGTACTTAAAGAAACGTGCAAAGAAGT } \\
\text { ATTAAAGGAACTCGAACAGTTAGAAAATAATCCAC-3' }\end{array}$ & $\begin{array}{l}\text { 5'-CATCTTTAAGAGCGATAGCTTCAAGTTCTATTGCT } \\
\text { ATTTGTAAAAGTGGATTATTITCTAACTGTTCGAG-3' }\end{array}$ \\
\hline R. massiliae & $\begin{array}{l}\text { 5'-CGTGCCGCAGTACTTAAAGAAACGTGCAAAGAAGT } \\
\text { ATTAAAGGAACTCGGGCAGTTAGACAACAATCCGC-3' }\end{array}$ & $\begin{array}{l}\text { 5'-CATCTTIAAGAGCGATAGCTTCAAGTTCTATTGCT } \\
\text { ATTTGTAAGAGCGGATTGTTGTCTAACTGCCCGAG-3' }\end{array}$ \\
\hline R. felis 1 & $\begin{array}{l}\text { 5'-CGTGCCGCAGTACTTAAAGAAACCTGCAAAGAAGT } \\
\text { ATTAAAGGAACTTGGACAGCTAGAAAACAACCCAC-3' }\end{array}$ & $\begin{array}{l}\text { 5'-CATCTTTAAGAGCGATAGCTTCAAGTTCTATTGCT } \\
\text { ATTTGCAAGAGTGGGTTGTTTCTAGCTGTCCAAG-3' }\end{array}$ \\
\hline R. felis 2 & $\begin{array}{l}\text { 5'-CGTGCCGCAGTACTTAAAGAAACTTGCAAAGAAGT } \\
\text { ATTAAAGGAACTCGGACAGCTAGAAAACAATCCGC-3' }\end{array}$ & $\begin{array}{l}\text { 5'-CATCTTAAGAGCGATAGCTTCAAGTTCTATTGCT } \\
\text { ATTTGCAAAAGCGGATTGTTTCTAGCTGTCCGAG-3' }\end{array}$ \\
\hline
\end{tabular}


processed as described above. Amplification products showing no or weak bands only in gel electrophoresis were re-amplified before further processing with actual real-time pyrosequencing. In each run, the $R$. helvetica plasmid was included as positive control.

\section{Statistical comparison of sequencing methods}

Chi-square test was performed to test the null hypothesis of no sensitivity differences between custom Sanger sequencing and real-time pyrosequencing. Sensitivity was calculated for all tick samples as well as tick samples divided into gltA copy numbers in the initial template. $\mathrm{H} 0$ was rejected if $\mathrm{P} \leq 0.05$.

\section{Competing interests}

The authors declare that they have no competing interests.

\section{Authors' contributions}

CS conceived the study and designed the oligonucleotides. EJ and SS carried out the laboratory experiments. EJ and CS drafted the manuscript. All authors participated in data analysis, and read and approved the final manuscript.

\section{Acknowledgements}

The authors would like to thank Sabine Schicht for technical assistance.

Received: 8 June 2012 Accepted: 4 October 2012

Published: 18 October 2012

\section{References}

1. Dumler JS, Barbet AF, Bekker CP, Dasch GA, Palmer GH, Ray SC, Rikihisa Y, Rurangirwa FR: Reorganization of genera in the families Rickettsiaceae and Anaplasmataceae in the order Rickettsiales: unification of some species of Ehrlichia with Anaplasma, Cowdria with Ehrlichia and Ehrlichia with Neorickettsia, descriptions of six new species combinations and designation of Ehrlichia equi and 'HGE agent' as subjective synonyms of Ehrlichia phagocytophila. Int J Syst Evol Microbiol 2001, 51:2145-2165.

2. Walker DH, Paddock CD, Dumler JS: Emerging and re-emerging ticktransmitted rickettsial and ehrlichial infections. Med Clin North Am 2008, 92:1345-1361.

3. Parola P, Paddock CD, Raoult D: Tick-borne rickettsioses around the world: emerging diseases challenging old concepts. Clin Microbiol Rev 2005, 18:719-756.

4. Simser JA, Palmer AT, Fingerle V, Wilske B, Kurtti TJ, Munderloh UG: Rickettsia monacensis sp. nov., a spotted fever group Rickettsia, from ticks (Ixodes ricinus) collected in a European city park. Appl Environ Microbiol 2002, 68:4559-4566.

5. Dobler G, Wolfel R: Typhus and other rickettsioses: emerging infections in Germany. Dtsch Arztebl Int 2009, 106:348-354.

6. Hartelt $\mathrm{K}$, Oehme R, Frank H, Brockmann SO, Hassler D, Kimmig P. Pathogens and symbionts in ticks: prevalence of Anaplasma phagocytophilum (Ehrlichia sp.), Wolbachia sp., Rickettsia sp., and Babesia sp. in Southern Germany. Int J Med Microbiol 2004, 293(Suppl 37):86-92.

7. Wolfel R, Terzioglu R, Kiessling J, Wilhelm S, Essbauer S, Pfeffer M, Dobler G: Rickettsia spp. in Ixodes ricinus ticks in Bavaria, Germany. Ann N Y Acad Sci 2006, 1078:509-511.

8. Fournier PE, Allombert C, Supputamongkol Y, Caruso G, Brouqui P, Raoult D: Aneruptive fever associated with antibodies to Rickettsia helvetica in Europe and Thailand. J Clin Microbiol 2004, 42:816-818.

9. Nilsson K, Lindquist O, Pahlson C: Association of Rickettsia helvetica with chronic perimyocarditis in sudden cardiac death. Lancet 1999, 354:1169-1173.

10. Nilsson K, Elfving K, Pahlson C: Rickettsia helvetica in patient with meningitis, Sweden, 2006. Emerg Infect Dis 2010, 16:490-492.

11. Boretti FS, Perreten A, Meli ML, Cattori V, Willi B, Wengi N, Hornok S, Honegger H, Hegglin D, Woelfel R, Reusch CE, Lutz H, Hofmann-Lehmann R:
Molecular Investigations of Rickettsia helvetica infection in dogs, foxes, humans, and Ixodes ticks. Appl Environ Microbiol 2009, 75:3230-3237.

12. Schicht S, Schnieder T, Strube C: Rickettsia spp. and coinfections with other pathogenic microorganisms in hard ticks from Northern Germany. J Med Entomol 2012, 49:766-771.

13. Burgdorfer W, Hayes SF, Mavros AJ: Nonpathogenic rickettsiae in Dermacentor andersoni: a limiting factor for the distribution of Rickettsia rickettsii. In Rickettsiae and rickettsial diseases. Edited by Burgdorfer W, Anacker RL. New York: Academic; 1981:585-594.

\section{doi:10.1186/1471-2334-12-261}

Cite this article as: Janecek et al:: SNP-based real-time pyrosequencing as a sensitive and specific tool for identification and differentiation of Rickettsia species in Ixodes ricinus ticks. BMC Infectious Diseases 2012 12:261.

\section{Submit your next manuscript to BioMed Central and take full advantage of:}

- Convenient online submission

- Thorough peer review

- No space constraints or color figure charges

- Immediate publication on acceptance

- Inclusion in PubMed, CAS, Scopus and Google Scholar

- Research which is freely available for redistribution 